Tohoku J. exp. Med., 1966, 90, 77-96

\title{
Derivation of Expiratory Mechanics of Normal and Emphysematous Lungs on Anatomical Basis
}

\author{
Norio Suwa, Katsuro Takahashi, Ryuitsu Fujimoto \\ and Yasuhiko Sasaki \\ The First Department of Pathology (Prof. N. Suwa), \\ Tohoku University School of Medicine, Sendai
}

\begin{abstract}
Mathematical derivations were discussed to predict expiratory efficiencies of individual autopsied lungs, normal and emphysematous, on anatomical basis. The following conditions were used in the derivation.

1) The contractile force of the lung is generated by the stress of a linear elastic system of randomized orientation. The stress function is of the form of $F=e^{a x}-1$, in which $F$ is the stress; $x$, the strain; and $a$, elasticity constant.

2) Geometrically similar configuration of the elastic system is approximately sustained irrespective of pulmonary expansion.

3) In the course of expiration intrapulmonary pressure is always equalized over the whole lung on account of intrapulmonary air flow.

4) The distribution of the density of the elastic system can be approximated to a combination of a small number $n$ of different normal distributions.

The results could be summarized as:
\end{abstract}

$$
\begin{aligned}
& V=\frac{P}{\sqrt{2} \pi} \sum_{j=1}^{n} \frac{V_{0 j}}{\sigma} \int_{0}^{\infty} \frac{(1+x)^{4}\left[(a x+a-2) e^{a x}+2\right]}{\left(e^{a x}-1\right)^{2}} \exp \left[-\frac{\left\{\frac{P(1+x)^{2}}{e^{a x}-1}-E_{j}\right\}^{2}}{2 \sigma_{j}^{2}}\right] d x \\
& \text { and } \frac{d t}{d P}=-\frac{3 R}{\sqrt{2 \pi} P} \sum_{j=1}^{n} \frac{V_{0 j}}{\sigma_{j}} \int_{0}^{\infty} \frac{(1+x)^{4}}{e^{a x}-1} \exp \left[-\frac{\left\{\frac{P(1+x)^{2}}{e^{a x}-1}-E_{j}\right\}^{2}}{2 \sigma_{j}^{2}}\right] d x,
\end{aligned}
$$

in which $V_{0 j}$ was partial pulmonary volume in relaxation; $E_{0 j}$, the arithmetical mean of the density of elastic system belonging to each normal distribution; $\sigma_{j}$, standard deviation of the latter; $R$, total airway resistance; $V$, total pulmonary volume at the pressure $P$; and $t$, time.

The first expression defined the pressure-volume relation of the lung and could also be used to obtain the initial pressure of expiration for a given pulmonary volume. The second gave the pressure-time relation in the expiratory phase. The combination of the two made possible the determination of expired volume in reference to time, with which the expiratory efficiency could be evaluated.

The obstructive expiratory distress in chronic pulmonary emphysema is generally attributed to the check valve mechanism of the bronchi. The concept of the mechanism was first explored and introduced by Dayman ${ }^{1}$ in 1951 into

Received for publication, May 2, 1966. 
clinical investigations of emphysema and has been supported by a majosity of clinicians with only slight modifications. The collapse of intrapulmonary airways, which plays an essential part in the mechanism, can take place only when the lung is compressed by an extraneous force. The condition is brought about in forced expiration, where expiration proceeds with the aid of overwhelming thoracic contractile force. In such a situation, intrinsic retractive force of the lung does not cause any significant difference in the expiratory efficiency, provided that bronchial walls are rigid enough to resist transmural pressure exerted upon them. Possible deteriorations of the contractile activity of emphysematous pulmonary tissues are not properly disclosed. Consequently, it is impossible to correlate anatomical findings of emphysematous lungs with the issue of the clinical tests for 'pulmonary functions' which are unfortunately performed mostly under forced expiration. Pathologists have after all little information about the expiratory function of the organ they observe at autopsy.

In the present report, an attempt was made to derive expiratory mechanics on the basis of anatomical findings of the lung. The results made it possible to predict respiratory efficiency of individual autopsied lungs independent of clinical function tests and were useful for analytical treatments of pulmonary expiratory functions.

\section{Mathematrcal Derivations}

\section{Intrapulmonary Pressure as a Function of Pulmonary Expansion}

Mathematical derivations are started from the following premises.

1) The lung occupies a certain limited volume in an infinitely large spatial extension of an elastic system.

2) The elastic system is constituted by linear structures of randomized orientation in the space. The contractile force of the lung is generated by the stress of the elastic system.

3) In a certain volume of adequate dimension, geometrically similar configuration of the elastic system is approximately sustained throughout pulmonary expansion and retraction.

4) Pulmonary expansion and contraction are reversible.

Some brief comments are necessary to the above premises. Since the lung is coated with the pulmonary pleura of entirely different elastic property from that of pulmonary tissues, mathematical treatments for intrapulmonary pressure should comprise a consideration on pleural effects. However, this is not easy because the configuration of the lung is not simply to assimilate to an analytically definable three-dimensional body. In the present study, pleural interference is not dealt with as an independent factor, but will be included in the treatment of pulmonary tissues. This principle is expressed in the first premise. 
The second premise implies that pulmonary contractile force is practically determined by intrapulmonary elastic fibers. Surface tension is not taken into consideration as an independent factor. This is because an expiration from the maximum pulmonary expansion is to be diszussed in the present study. Under this condition, the contribution of surface tension is practically negligible.

The third premise is evidently not strictly satisfied particularly in emphysematous lungs where the density of the elastic system is remarkably different in different pulmonary portions. It is impossible to maintain geometrically similar configurations over the total lung without separating pulmonary portions with different density of the elastic system from one another. With actual lungs in which the continuity of tissues must be sustained irrespective of pulmonary expansion, some errors are inevitable. We must be content with a practical approximation.

The last premise is a requirement for the preservation of free mechanical energy of the system. The energy stored in the system by pulmonary expansion is perfectly released and utilized to drive air out of the lung.
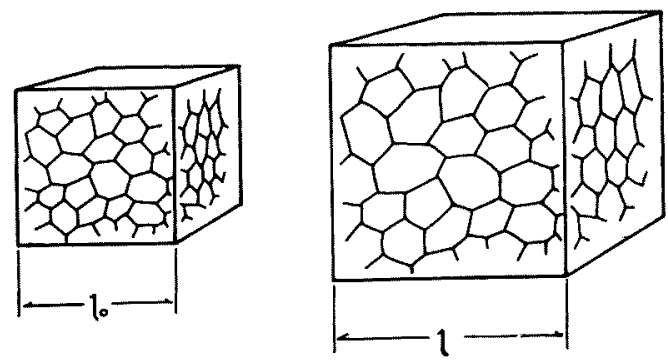

$$
x=\frac{1-10}{10}
$$

Fig. 1. A cube with edges of $l_{0}$ in length is taken in a relaxed lung and is supposed to be uniformly expanded to a state in which $l_{0}$ is stretched to $l$. No restriction is necessary to the geometrical configuration of alveolar septa or elastic system, except that it must be similar to each other in two conditions. Nor is it required that the configuration of alveoli is assimilated to a certain analytically definable three-dimensional body.

Suppose now a cube with edges of $l_{0}$ in length in a perfectly relaxed lung and expand it uniformly in every direction so that its edges attain a length of $l$. The total length of the elastic system, the total surface area of alveolar septa and the volume of the cube in relaxed and expanded states are designated as $L_{0}$. $S_{0}$ and $V_{0}$; and $L, S$ and $V$, respectively. If $x$ is defined by the following form:

$$
\left(l-l_{0}\right) / l_{0}=x
$$

it is a dimensionless variable indicating the strain. The quantity is equivalent 
to the rate of pulmonary stretch in length and is hereafter simply called the 'stretch' or 'extension' of the lung.

We can now express $L, S$ and $V$, and their differentials with $x$ as follows:

$$
\begin{array}{ll}
L=L_{0}(1+x) & d L=L_{0} d x \\
S=S_{0}(1+x)^{2} & d S=2 S_{0}(1+x) d x \\
V=V_{0}(1+x)^{3} & d V=3 V_{0}(1+x)^{2} d x .
\end{array}
$$

If we suppose that the lung is further expanded from this state by a minute stretch $d x$ and the minute work necessary for the expansion is $d W$, the latter is defined by:

$$
d W=F d L=T d S=P d V
$$

in which $F, T$ and $P$ are stress of the elastic system, tension on the alveolar surface and intrapulmonary pressure, respectively. By substitution of $d L, d S$ and $d V$ with (1), we obtain:

$$
d W=F L_{0} d x=2 T S_{0}(1+x) d x=3 P V_{0}(1+x)^{2} d x,
$$

and hence:

$$
P=\frac{L_{0}}{3 V_{0}} \cdot \frac{F}{(1+x)^{2}}
$$

We assume that $F$ is given by the stress function proposed in a previous report of ours ${ }^{2}$ in the form of:

$$
F=A\left(e^{a . r}-1\right) .
$$

Because $A$ is a constant determined by the quantity of the elastic system and can be included in $L_{0}$, we write henceforth simply:

$$
F=e^{a x}-1 \text {. }
$$

If $\rho_{0}$ is the length of the elastic system on a unit surface area of alveolar septa, $L_{0}$ is written as:

$$
L_{0}=\rho_{0} S_{0}
$$

By (4) and (5), the expression (3) is now:

$$
P=\frac{\rho_{0} S_{0}}{3} \cdot \frac{e^{a x}-1}{(1+x)^{2}}
$$

and by further substitution of $\rho_{0} S_{0} / 3 V_{0}$ by $E$, the final result is:

$$
P=E \frac{e^{a x}-1}{(1+x)^{2}}
$$

The expression (7) defines intrapulmonary pressure as a function of pulmonary extension and consequently of pulmonary volume, as the latter is 
given by $V=V_{0}(l+x)^{3}$. Since this is the most fundamental expression in pulmonary mechanics, it would be adequate to comment on its implications. The constant $E$ is equal to a third of the density of the pulmonary elastic system, as is indicated by (3) and (6). Consequently, it may simply be regarded as representing intrapulmonary density of the elastic system, so far as comparative studies of different lungs are concerned. It is thus expressed by (7), that intrapulmonary pressure is proportional to the stress and density of the pulmonary elastic system and inversely proportional to the square of pulmonary extension. The latter relation comes from the reduction in the density of the elastic system with increasing pulmonary expansion.

The derivation of (7) is likewise possible in an entirely different way. The intrapulmonary pressure is equal to the sum of the normal components of forces exerted on a unit surface area of an imaginary plane. Accordingly, an approach to the problem can be made as follows. Consider a lung in a state of expansion and take a cube of unit volume in it. If the stretch of the lung is $x$, the length of the elastic system in the cube is given by:

$$
L / V=L_{0}(1+x) / V_{0}(1+x)^{3}=L_{0} / V_{0}(1+x)^{2} .
$$

The elastic system in the cube is then considered to be divided into an infinitely large number $n$ of infinitesimally small fractions of $\eta$ in length. In this case:

$$
L_{0} / V_{0}(1+x)^{2}=n \eta
$$

by definition. The fractions are now supposed to be displaced without changing their directions in the space to a certain point $O$ in the cube, so that the middle point of each fraction is situated on $O$. The fractions assembled in this way fill a sphere of $\eta / 2$ in raidus with an equal density in every direction. Take now an imaginary plane across the cube parallel to one of its faces. The surface area of the plane is equal to a unit and is accordingly 1 . The expected value for a fraction with an angle of inclination $\theta$ to the imaginary plane to cross the plane is now designated as $\varepsilon_{\theta}$. This is given when the effective length of the fraction in the normal direction to the plane is divided by the length of an edge of the cube. Because the cube is of a unit volume the length of its edges is 1. Accordingly, the result is simply:

$$
\varepsilon_{\theta}=\eta \sin \theta
$$

The number of fractions $d n_{\theta}$ with angles of inclination to the plane between $\theta$ and $\theta+d \theta$ is proportional to the surface area of a minute belt made by the fractions of the above range on a hemisphere of the sphere made by the total fractions. Consequently:

$$
\begin{aligned}
d n_{\theta} & =n \frac{2 \pi(\eta / 2)^{2} \cos \theta}{2 \pi(\eta / 2)^{2}} d \theta \\
& =n \cdot \cos \theta d \theta
\end{aligned}
$$




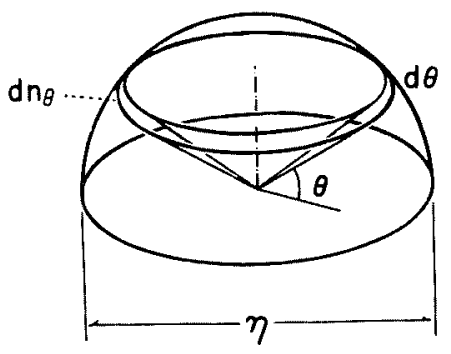

$d n \theta=n \cdot \cos \theta \cdot d \theta$

Fig. 2. The hemisphere in the diagram is supposed to be made by fractions of the elastic system assembled to a point. When the orientation of the elastic system is completely randomized in the space, the density of the fractions is uniform in every direction, and the number of fractions is proportional to the surface area made by the same fractions on the surface of the hemisphere.

The expected value $d C_{\theta}$ for the fractions of the above range to cross the imaginary plane is given by the product of $\varepsilon_{\theta}$ and $d n_{\theta}$ as:

$$
\begin{aligned}
d C_{\theta} & =\varepsilon_{\theta} \cdot d n_{\theta} \\
& =n \eta \cdot \sin \theta \cos \theta \cdot d \theta \\
& =-\frac{L_{0}}{V_{0}(1+x)^{2}} \sin \theta \cos \theta \cdot d \theta .
\end{aligned}
$$

This indicates at the same time the number of fibers of the elastic system which cross the imaginary plane with angles of inclination of the above range.

The normal component $d P_{\theta}$ of the force exerted upon the plane by the fibers of the above range is given accordingly by:

$$
d P_{\theta}=\frac{F L_{0}}{V_{0}(1+x)^{2}} \cdot \sin ^{2} \theta \cos \theta \cdot d \theta
$$

in which $F$ is the stress of individual fibers of the elastic system. The pressure $P$ on the imaginary plane is the sum of the normal components of forces contributed by fiber groups of every possible inclination on a unit surface area. Consequently, expression (8) is integrated and the result gives the intrapulmonary pressure $P$ as:

$$
\begin{aligned}
P & =\frac{F L_{0}}{V_{0}(1+x)^{2}} \int_{0}^{\pi / 2} \sin ^{2} \theta \cos \theta \cdot d \theta \\
& =\frac{F L_{0}}{3 V_{0}(1+x)^{2}}\left[\sin ^{3} \theta\right]_{0}^{\pi / 2} \\
& =\frac{F L_{0}}{3 V_{0}(1+x)^{2}} .
\end{aligned}
$$


This is exactly the expression (3) which was derived from the principle of preservation of mechanical energy.

We can now proseed to examine whether the pressure-volume relation defined by (7) is in good agreement with clinical observations. For the calculation of (7) the lung volume in relaxation $V_{0}$ must be known. Direct measurement of the quantity is unexpectedly difficult. The total volume of normal lungs of young adults estimated by water replacement immediately after the organs were excised from thoracic cavities does not exceed $800 \mathrm{~cm}^{3}$. In this result $V_{0}$ is evidently underestimated because a considerable number of alveoli is collapsed and apparently closed. When the same lungs are expanded by intrabronchial infusion of physiological saline, floated on water and allowed to contract with their own retractive force, the lung volumes in 'relaxation' are about 1,200 $\mathrm{cm}^{3}$ or more. These values are in all probability too high, because the lung can not overcome its internal friction under a certain limit of its expansion. However, since a correct value for the lung volume in relaxation would not be much different from $1,000 \mathrm{~cm}^{3}$ in any way, $V_{0}$ is assumed to be $1,000 \mathrm{~cm}^{3}$ for healthy young adults in the subsequent derivations.

The elasticity constant $a$ for the normal lung is regarded to be 5.5 on the basis of our previous investigation.

For the calculation of (7) it is further necessary to determine the value of $E$. In the present situation, however, the estimation would not be easy unless we have recourse to clinical observations. At a lung volume of about $2,500 \mathrm{~cm}^{3}$ which corresponds to FRC (functional residual capacity), intrapulmonary pressure is approximately $5 \mathrm{~cm} \mathrm{H}_{2} \mathrm{O}$. From this result, $E$ was estimated as 1.5 . The lung is supposed to be expanded with normal tidal volume of $500 \mathrm{~cm}^{3}$ and further to the maximum volume or TLC (total lung capacity), which is usually estimated from $5,000 \mathrm{~cm}^{3}$ to $5,500 \mathrm{~cm}^{3}$ for normal Japanese male adults. Intrapulmonary pressure was calculated with (7) at pulmonary volumes of $3,000 \mathrm{~cm}^{3}, 5,000 \mathrm{~cm}^{3}$ and $5,500 \mathrm{~cm}^{3}$, and is given in Table 1 . The results are in satisfactory agreement with clinical estimations.

TABLE 1

\begin{tabular}{|c|c|c|}
\hline \multicolumn{2}{|c|}{ Lung volume $\left(\mathrm{cm}^{3}\right)$} & Pressure $\left(\mathrm{cm} \mathrm{H}_{2} \mathrm{O}\right.$ ) \\
\hline FRC & 2,500 & 5.0 \\
\hline $\mathrm{FRC}+\mathrm{TV}$ & 3,000 & 7.5 \\
\hline TLC & 5,000 & 25.0 \\
\hline & 5,500 & 31.9 \\
\hline
\end{tabular}

The calculation was performed with the following values for the parameters:

$$
\begin{array}{ccc}
V_{0}=1,000 \mathrm{~cm}^{3} & a=5.5 & E=1.5 . \\
\text { FRC: Functional residual capacity. } & \mathrm{TV}: \text { Tidal volume. }
\end{array}
$$

TLC: Total lung capacity. 


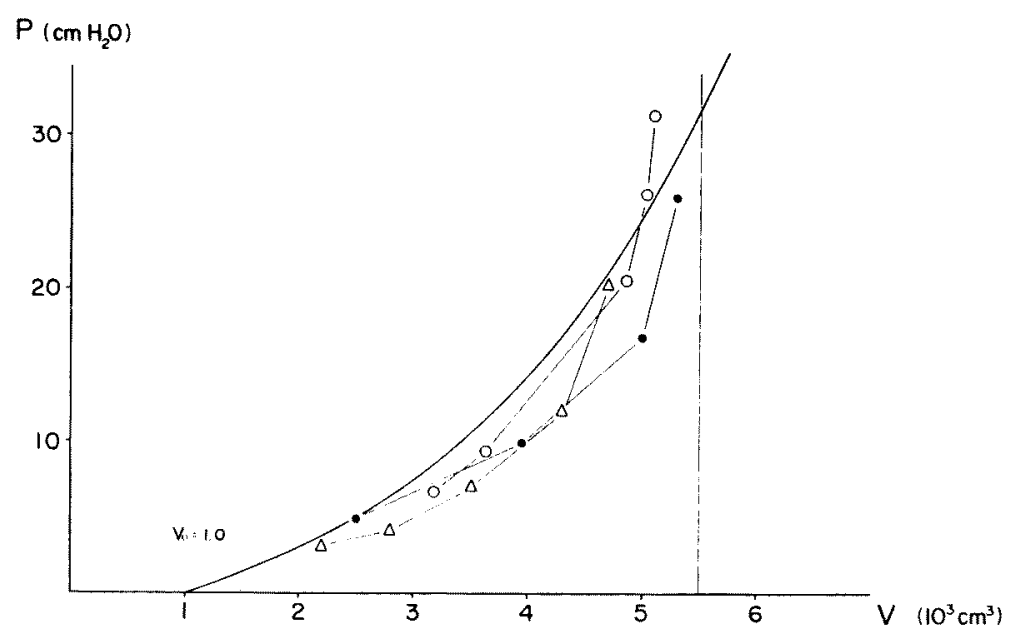

Fig. 3. A theoretical curve for the volume-pressure relation of the normal adult lung is correlated to a number of clinical observations in expiratory phase. (By courtesy of Dr. Takishima).

In Fig. 3, the pattern of the curve given by (7) is correlated to a number of clinical measurements in the expiratory phase. Accelerated elevation of intrapulmonary pressure with increasing pulmonary volume, which is characteristic of the volume-pressure relation of the lung, is sufficiently reproduced by (7).

\section{Expiratory Dynamics}

In clinical investigations under forced expiration, the effect of turbulent flow is usually taken into consideration. ${ }^{3}$ The relation of pressure and flow is generally given by:

$$
P=K_{1}\left(\frac{d V}{d t}\right)+K_{2}\left(\frac{d V}{d t}\right)^{2},
$$

in which $K_{1}$ and $K_{2}$ are constants and the effect of turbulent flow is expressed in the term $K_{2}(d V / d t)^{2}$. When expiration proceeds with the contractile force of the lung itself, the second term in the above expression becomes much less significant. In the present study, intrabronchial air flow is assumed to be laminar in every phase of expiration, and the effect of turbulent flow is neglected.

If the total airway resistance is $R$, we obtain from Hagen-Poiseuille's law:

$$
-\frac{d V}{d t}=\frac{P}{R}
$$

The sign of minus indicates the expiratory phase, where pulmonary volume decreases with the lapse of time $t$. Our next purpose is to derive a differential equation which gives a pressure-time relation in the expiratory phase. The 
derivative $d t / d P$ is transformed as follows:

$$
\frac{d t}{d P}=\frac{d V}{d P} \cdot \frac{d t}{d V}=\frac{d V}{d x} \cdot \frac{d x}{d P} \cdot \frac{d t}{d V}
$$

From (1), (7) and (9), we obtain:

$$
\begin{aligned}
& \frac{d V}{d x}=3 V_{0}(1+x)^{2} \\
& \frac{d x}{d P}=\frac{(1+x)^{3}}{E\left[(a x+a-2) e^{a x}+2\right]}=\frac{\left(e^{a x}-1\right)(1+x)}{P\left[(a x+a-2) e^{a x}+2\right]} \\
& \frac{d t}{d V}=-\frac{R}{P},
\end{aligned}
$$

and consequently from (10):

$$
\frac{d t}{d P}=-\frac{3 R V_{0}}{P^{2}} \cdot \frac{\left(e^{a x}-1\right)(1+x)^{3}}{(a x+a-2) e^{a x}+2} .
$$

The equation apparently includes $x$ as a variable, but $x$ is in reality a function of $P$ determined by (7), and consequently the right side of the equation (11) has $P$ as the single variable. An analytical treatment of the equation (11) is not possible,

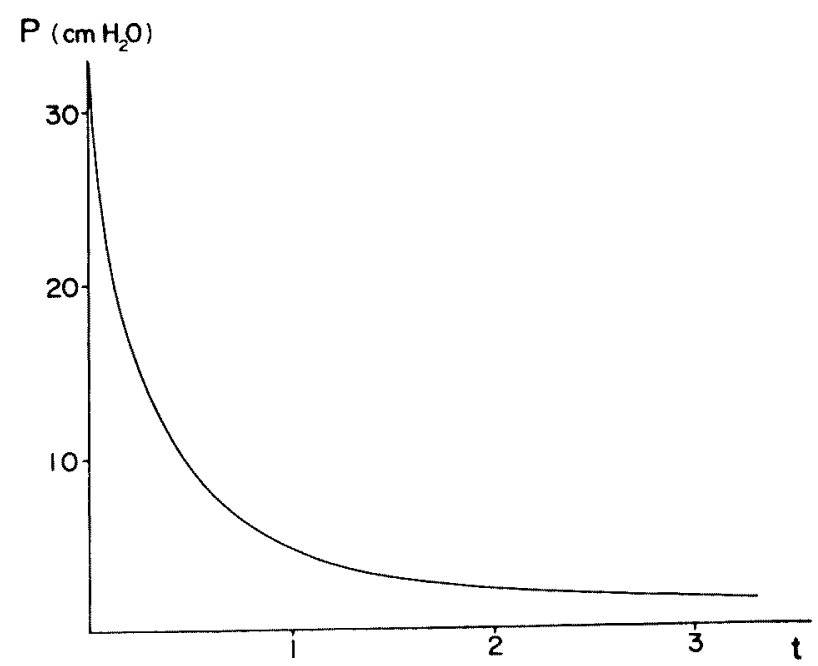

Fig. 4. An example of the time-pressure relation is demonstrated based on the calculation of (11) for expiration from a lung volume of $5,500 \mathrm{~cm}^{3}$. The determination of the time scale is to be discussed in a forthcoming report. In the present report, it is to be regarded merely as an arbitrarily selected interval. 


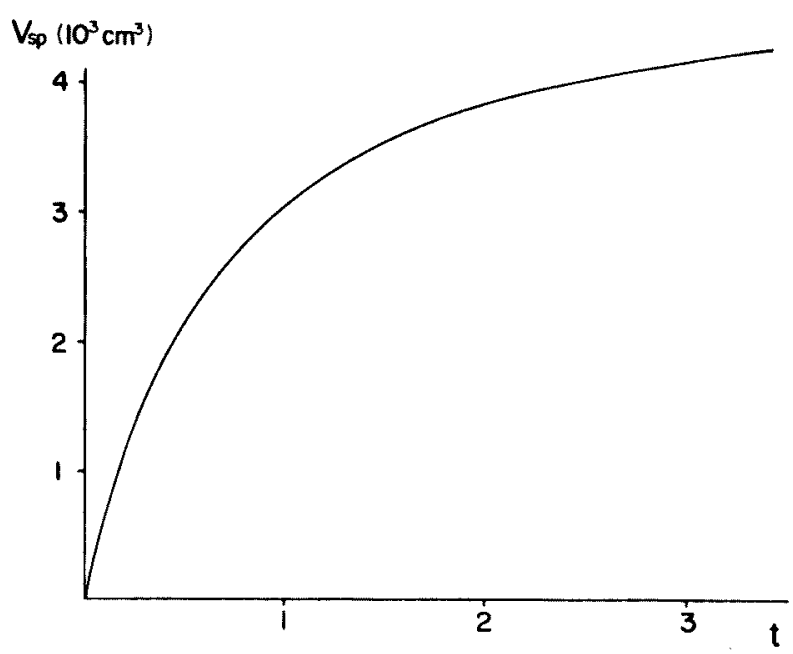

Fig. 5. The time-expired volume relation is presented with the same pulmonary model as in Fig. 4.

but the curve represented by it can be determined by means of an electronic computer with desired accuracy. In Fig. 4 an example of the curve is presented. When time is correlated to pulmonary volume by way of (11), (7) and (1), the volume of expired air $V_{s p}$ can be calculated in reference to time as in Fig. 5.

The equation (11) is derived on the assumption that the density of elastic system is uniform throughout the lung. In the treatment of emphysematous lungs, however, which are characterized by highly uneven distribution of elastic system, some modification of (11) is of course necessary. We start from a simple model in which the lung is composed of two parts with different density of elastic system. The two parts are assumed to be of the same volume of 500 $\mathrm{cm}^{3}$ in relaxation, and in one of them the density of elastic system is 1 and in the other, 1/10. The former is to represent normal, the latter, emphysematous, pulmonary tissue. When the model is expanded, the inflation of the emphysematous part is much larger than that of the normal part. At the pulmonary volume of $5,500 \mathrm{~cm}^{3}$, the emphysematous part oxcupies about $70 \%$ of the total lung volume instead of $50 \%$ in relaxation. This means that in emphysematous lungs the greater part of inspired air is taken up in pathological pulmonary portions, and the expansion of the normal part is seriously suppressed. This is of course one of the disadvantages of emphysematous lungs in respiration.

Now, in the expiration of a lung with uneven distribution of the elastic system, two types of expiration must be distinguished. The one takes place when there is no intrapulmonary communication for air except for the 

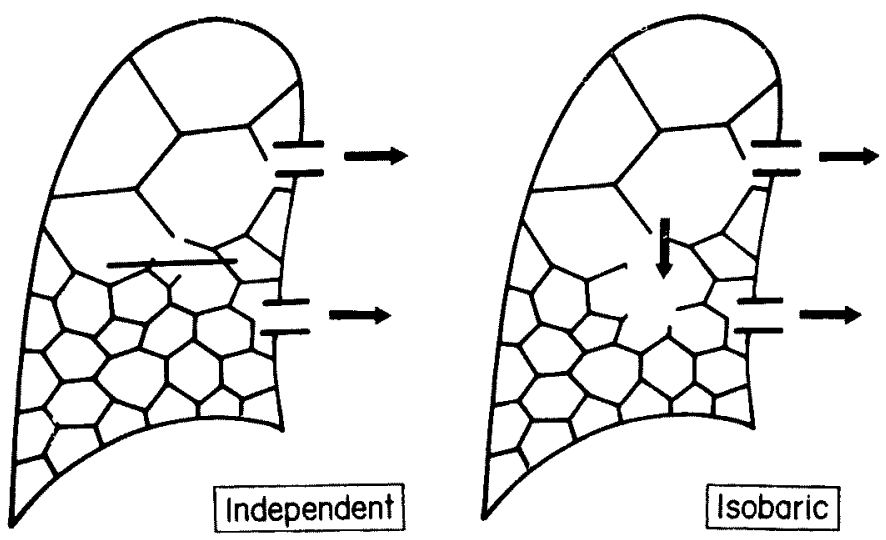

Fig. 6. The distinction of independent and isobaric expiration is diagrammatically demonstrated. The expiration of emphysematous lung is obviously very close to the isobaric expiration on account of abundant intrapulmonary communications.

bronchi. At the beginning of expiration intrapulmonary pressure is equal throughout the lung, but with the advancement of expiration pressure drop in the part with low density of the elastic system is retarded. As a result, expiration proceeds under different intrapulmonary pressure in different parts of the lung to its termination after an infinitely long time. Throughout the course the pressure in the part with low density of the elastic system is always higher than in the part with high density of the system. The expiration of this type in which individual parts of the lung contract without interference to one another is called 'independent' expiration in the subsequent part of this report.

The other type of expiration is associated with a condition of the lung where abundant intrapulmonary pathways for air are present, so that intrapulmonary pressure difference is brought to equilibrium in every phase of expiration. Intrapulmonary pressure is equal over the whole lung throughout the expiration. The expiration of this type is called 'isobaric' expiration, and the intrapulmonary air flow by which intrapulmonary pressure is equalized is designated as 'transalveolar flow.' In the lungs of emphysema, extensive destruction of alveolar walls provides unobstructed passage to air among different pulmonary parts. It is evident that the expiration of emphysematous lungs is practically of the type of isobaric expiration. Consequently, our next step is to derive a differential equation of isobaric expiration for lungs with uneven density of the elastic system.

This is possible through purely mathematical operations which are analogous to those in the derivation of (11). However, it is better to derive the equation on the basis of the physical processes involved in the isobaric expiration. 


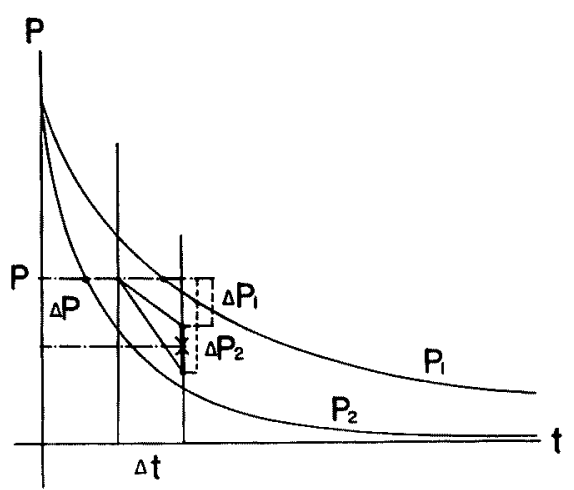

Fig. 7. Physical processes involved in the derivation of the differential equation for isobaric expiration are presented. Two curves $P_{1}$ and $P_{2}$ in the diagram represent the time-pressure relations in independent expirations of the two pulmonary parts with different density of the elastic system. Independent expirations of the two parts for a very short time $\Delta t$ from a common pressure $P$ are demonstrated by two divergent short straight lines, which are parallel to the tangents (not given in the diagram) of the curves $P_{1}$ and $P_{2}$ at pressure $P$, respectively. Intrapulmonary pressure difference after the independent expirations of individual pulmonary parts for $\Delta t$ is brought to equilibrium without any lapse of time. The pressure difference $\Delta P$ from $P$ when intrapulmonary pressure is equalized and the short lapse of time $\Delta t$ give the pressure gradient for the differential equation of isobaric expiration.

Suppose that the lung is composed of $n$ parts with different density of elastic system. The volume of individual parts at pressure $P$ is expressed by $V_{i}$. We assume now, that the lung has contracted from a certain given total lung volume in isobaric expiration to the point of pressure $P$ and time $t$ and is allowed further to contract isobarically for a very short time $\Delta t$, bringing about an pressure increase of $\Delta P$. In reality, the pressure drops with time and $\Delta P$ is always negative. This short isobaric expiration can be divided into two steps. In the first step, individual pulmonary parts are allowed to contract independently. Because these parts are all different in their density of the elastic system, intrapulmonary pressure at the end of the independent expiration is different in all the pulmonary parts. In the second step, the pressure in the lung is brought to equilibrium on account of transalveolar flow. When intrapulmonary communications are abundant and the resistance to air flow can be regarded practically as zero, there is no appreciable lapse of time in the course of pressure equalization. Accordingly, the air does not flow out of the lung during pressure equalization by way of the bronchi with finite resistance, and the total lung volume remains unchanged. The relation can be thus expressed as:

$$
\sum_{i=1}^{n} \frac{\partial V_{i}}{\partial P} \Delta P=\sum_{i=1}^{n} \frac{\partial V_{i}}{\partial t} \Delta t
$$


In this expression $V_{i}$ is regarded as a function determined by two variables $P$ and $t$, and the partial differential coefficient $\partial V_{i} \partial P$ is related to isobaric, and $\partial V_{i} / \partial t$ to independent, expiration. The equation indicates accordingly that the minute volume change of the total lung from a common pressure $P$ is the same in isobaric and independent expirations. The partial differential coefficients in (12) can be further transformed as follows:

$$
\begin{aligned}
& \sum_{i=1}^{n} \frac{\partial V_{i}}{\partial P}=\sum_{i=i}^{n} \frac{d x_{i}}{d P} \cdot \frac{d V_{i}}{d x_{i}}=\sum_{i=1}^{n} \frac{3 V_{0 i}\left(e^{a_{i} x_{i}}-1\right)\left(1+x_{i}\right)^{3}}{P\left[\left(a_{i} x_{i}+a_{i}-2\right) e^{a_{i} x_{i}}+2\right]} \\
& \sum_{i=1}^{n} \frac{\partial V_{i}}{\partial t}=-P \sum_{i=1}^{n} \frac{1}{R_{i}}=-\frac{P}{R}
\end{aligned}
$$

in which the quantities belonging to the pulmonary volume $V_{i}$ are indicated by the subscript $i$, and those for the total lung are without the subscript. The derivation of (14) is possible because airway resistance to individual parts of the lung is inserted in parallel. From (12), (13) and (14) we obtain the following equation, if $\Delta P$ and $\Delta t$ are taken infinitesimally small and written as $d P$ and $d t$ :

$$
\frac{d t}{d P}=-\frac{3 R}{P^{2}} \sum_{i=1}^{n} \frac{V_{0 i}\left(e^{a_{i} x_{i}}-1\right)\left(1+x_{i}\right)^{3}}{\left(a_{i} x_{i}+a_{i}-2\right) e^{a_{i} x_{i}}+2}
$$

in which $x_{i}$ is determined by:

$$
P=E_{i} \frac{e^{a_{i} x_{i}}-1}{\left(1+x_{i}\right)^{2}}
$$

and the equation (15) is given with $P$ as the single variable. From the comparison of (15) and (11), it is clear that (11) is a special form of (15). When $n$ is equal to 1 , or the lung is homogeneous in the density of its elastic system, (11) is immediately obtained from (15).

The next problem is to examine how the expiratory efficiency is different between the two types of expiration, isobaric and independent. We use again the simple model, in which the lung is composed of two parts with the densities of elastic system of 1 and 0.1 , respectively, and of equal volume of $500 \mathrm{~cm}^{3}$ in relaxation. When the lung is expanded to $5,500 \mathrm{~cm}^{3}$, intrapulmonary pressure is only about $8 \mathrm{~cm} \mathrm{H}_{2} \mathrm{O}$, which is still lower than a third of the pressure in the lung with the elastic system of uniform and normal density. Such a low initial pressure is of course one of the major causes for deteriorating the expiratory efficiency of emphysematous lungs. The curves of expiration are presented in Figs. 8 and 9. The result demonstrates an improvement, though not very marked, of expiratory efficiency in isobaric expiration. The effect depends upon the form of stress function. With the stress function employed in this investigation, expiratory efficiency is always higher in isobaric expiration. It is interesting 


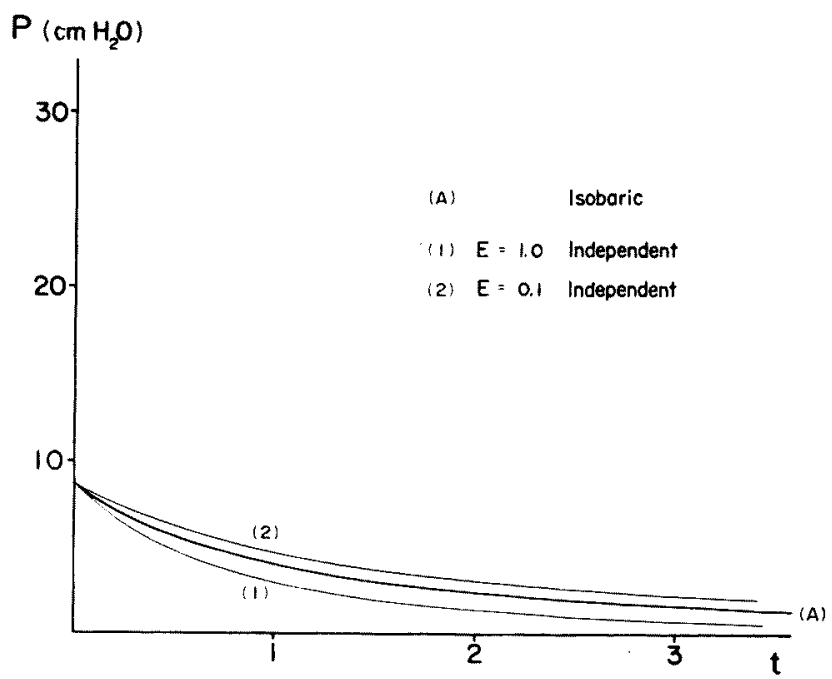

Fig. 8. The time-pressure relation in different types of expiration with a simple pulmonary model is demonstrated. The lung is assumed to be composed of two parts with densities of the elastic system of 1 and 0.1. Each of the two parts has an equal volume of $500 \mathrm{~cm}^{3}$ in relaxation. Note the low initial pressure at a total lung volume of $5,500 \mathrm{~cm}^{3}$. In the course of the independ. ent expiration the pressure level is always higher in the part of lower density of the elastic system.

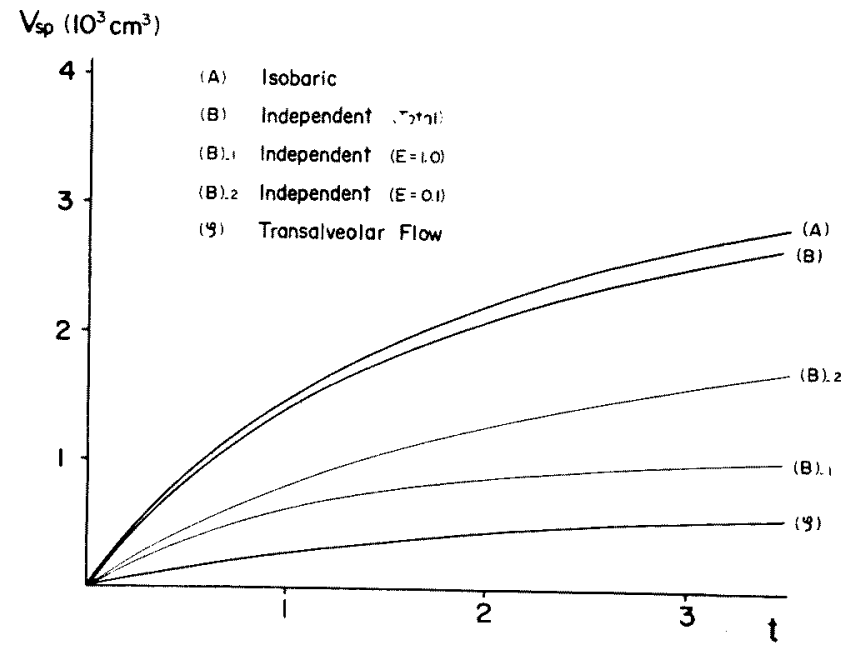

Fig. 9. Slight elevation in expiratory efficiency is observed in the isobaric expiration in comparison with the independent. The expired volume from the total lung in independent expiration is the sum of those from individual parts, which are indicated by two slender curves in the diagram. The transalveolar flow $\varphi$ attains about $20 \%$ of the expired volume in isobaric expiration at the point where the latter reaches to $3,000 \mathrm{~cm}^{3}$. The same pulmonary model is used as in Fig. 8. 
that destruction of alveolar walls, which is in its nature a distinctly pathological process, acts as a compensatory mechanism in expiratory mechanics of emphysematous lungs. Transalveolar flow in the model reaches to about $20 \%$ of expired volume at the point where the latter attains $3,000 \mathrm{~cm}^{3}$. This means that about $600 \mathrm{~cm}^{3}$ out of the total expired volume have once been driven from the emphysematous, into the normal, part and then leave the lung via preformed airways belonging to the normal part. It is evident that transalveolar flow ameliorates the disturbance of gas exchange in emphysema, since it provides a contact with normal alveolar septa for a considerable volume of air inspired in pulmonary tissues of reduced density.

\section{Transformation of the Differential Equation of Isobaric Expiration for Lungs in Which the Distribution of Elastic System is Given by a Continuous Function}

In the treatment of autopsied lungs it is necessary to transform the equation (15), so thct it can be applied to cases with a continuous distribution of the density of elastic system. The distribution of its density in normal and emphysematous lungs can sufficiently be approximated to a normal distribution or to a combination of a small number of different normal distributions. Since the problem is to be discussed more extensively in a forthcoming report of ours, we describe here only mathematical derivations in view of the above cases.

We suppose now that the distribution of elastic system is defined by a normal distribution with arithmetical mean $E$ and standard deviation $\sigma$. If the total lung volume in relaxation is $V_{0}$, the volume of pulmonary part in which the density of elastic system is between $E$ and $E+d E$ is given as $V_{O E} \cdot d E$, when $V_{O E}$ is determined by:

$$
V_{O E}=\frac{V_{0}}{\sqrt{2 \pi} \sigma} \exp \left[-\frac{(E-\bar{E})^{2}}{2 \sigma^{2}}\right]
$$

If the distribution of the elastic system must be approximated to a combination of $n$ different normal distributions, (16) is to be written as:

$$
V_{O E}=\frac{1}{\sqrt{2} \pi}=\sum_{j=1}^{n} \frac{V_{0 j}}{\sigma_{j}} \exp \left[-\frac{\left(E-\bar{E}_{j}\right)^{2}}{2 \sigma_{j}^{2}}\right]
$$

in which $\sum_{j=1}^{n} V_{0 j}=V_{0}$.

We assume now that all pulmonary parts have a common elasticity constant a. In a pulmonary model with a discontinuous distribution of elastic system, the pulmonary volume and the differential equation for isobaric expiration are:

$$
V=\sum_{i=1}^{n} V_{0 i}\left(1+x_{i}\right)^{3}
$$


and

$$
\frac{d t}{d P}=-\frac{3 R}{P^{2}} \sum_{i=1}^{n} \frac{V_{0 i}\left(e^{a x_{i}}-1\right)\left(1+x_{i}\right)^{3}}{\left(a x_{i}+a-2\right) e^{2 x_{i}}+2}
$$

respectively.

In (18) and (19), $V_{0 i}$ is the volume of individual pulmonary parts with different density of elastic system. Consequently, by substitution of $V_{0 i}$ with $V_{O E} \cdot d E$ and by replacing the sign of $\Sigma$ in (18) and (19) with the sign of integration, we can obtain a pressure-volume relation and a differential equation for isobaric expiration for lungs with continuous distribution of the density of the elastic system. In this procedure, the integration is more conveniently to perform with $d x$ than with $d E$. From the relation:

$$
E=P \frac{(1+x)^{2}}{e^{a x}-1}
$$

we obtain:

$$
d E=-\frac{P(1+x)\left[(a x+a-2) e^{a x}+2\right]}{\left(e^{a x}-1\right)^{2}} d x
$$

with which the integration with $d E$ can be converted to that with $d x$. Because $E$ and $x$ cannot take negative values, it is assumed that the integration of normal distributions from $-\infty$ to $+\infty$ is practically equal to that from 0 to $+\infty$. Further it is to be noticed that $x$ is zero when $E$ is infinitely large and $x$ is infinitely large when $E$ is zero. The expressions (18) and (19) are thus transformed to:

$$
\begin{array}{r}
V=\frac{P}{\sqrt{2} \pi} \sum_{j=1}^{n} \frac{V_{0 j}}{\sigma_{j}} \int_{0}^{\infty} \frac{(1+x)^{4}\left[(a x+a-2) e^{a x}+2\right]}{\left(e^{a x}-1\right)^{2}} \\
\times \exp \left[-\frac{\left\{\frac{P(1+x)^{2}}{e^{a x}-1}-\bar{E}_{j}\right\}^{2}}{2 \sigma_{j}^{2}}\right] d x
\end{array}
$$

and $\quad \frac{d t}{d P}=-\frac{3 R}{\sqrt{2 \pi} P} \sum_{j=1}^{n} \frac{V_{0 j}}{\sigma_{j}} \int_{0}^{\infty} \frac{(1+x)^{4}}{e^{a x}-1}$

$$
\times \exp \left[-\frac{\left\{\frac{P(1+x)^{2}}{e^{a x}-1}-\bar{E}_{j}\right\}^{2}}{2 \sigma_{j}^{2}}\right] d x .
$$

With these two expressions we are now enabled to calculate expiratory efficiencies of individual autopsied lungs. When we give to $V$ a value larger than $V_{0}$ and determine $P$ from (20), we obtain the initial condition for the differential equation (21). The solution of (21) gives a pressure-time, and in combination with (20), an expired volume-time, relation for the expiration from a lung volume $V$. Analytical solution of (20) and (21) is obviously impossible and 
we must use an electronic computer for the determination of the curves represented by the two expressions.

\section{Transalveolar Flow}

The present report is concluded with the determination of transalveolar flow or of the intrapulmonary air flow in the course of an isobaric expiration. We assume again that the isobaric expiration for a very short time is divided into two steps just as in the derivation of (12). In the first step, individual pulmonary parts are allowed to contract independently. Intrapulmonary pressure difference produced in the first step is then brought to equilibrium in the second step on account of transalveolar flow. In the latter step, intrapulmonary air flow takes place always from the part of low density, to the part of high density, of the elastic system. When the resistance to intrapulmonary air flow is zero, the total lung volume remains the same in spite of intrapulmonary air flow. Consequently, the part with low density of the elastic system contracts, and the part with high density of the system expands and receives the quantity of air expelled from the former part. If the distribution of the density of the elastic system is given by

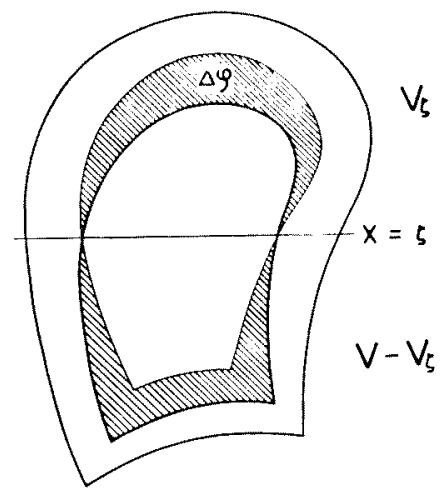

Fig. 10. The outermost line represents the pulmonary volume at a pressure $P$. A further isobaric expiration for a very short time interval $\Delta t$ brings the pulmonary volume to the state enclosed by a thick line in the diagram. This process is supposed to be composed of two steps. In the first step, the lung contracts in independent expiration to the state delineated by a slender line. In the second step pressure equalization takes place on account of minute transalveolar flow $\Delta \Phi$ (shadowed in the diagram)," and the lung comes to the final state corresponding to the end of the isobaric expiration for $\Delta t$. The total lung volume remains unchanged in the course of pressure equalization. When the distribution of the elastic system is defined by a continuous function, there must be some parts in the lung, which neither contract nor expand in the second step. The 'stretch' $x$ of these parts at the pressure $P$ is designated as $\zeta$. It is not required, however, that these parts are assembled in a spatially continuous place as in the diagram. 
a continuous function, there must be a pulmonary part which neither contracts nor expands in the course of pressure equalization.

We designate the density of the elastic system of this part as $E_{\zeta}$ and its stretch or $x$ at pressure $P$ as $\zeta$. When we suppose a lung with a homogeneous density $E_{\zeta}$ of the elastic system, the derivative $d t / d P$ of this lung must be equal to that of isobaric expiration in the case with uneven distribution of the density of the elastic system. Accordingly, we obtain:

$$
\begin{aligned}
\frac{d t}{d P} & =-\frac{3 R V_{0}}{P^{2}} \cdot \frac{\left(e^{2 \zeta}-1\right)(1+\zeta)^{3}}{(a \zeta+a-2) e^{a \zeta}+2} \\
& =-\frac{3 R}{\sqrt{2} \frac{R}{\pi} \cdot P} \sum_{j=1}^{n} \frac{V_{0 j}}{\sigma_{j}} \int_{0}^{\infty} \frac{(1+x)^{4}}{e^{a x}-1} \exp \left[-\frac{\left\{\frac{P(1+x)^{2}}{e^{a x}-1}-\bar{E}_{j}\right\}^{2}}{2 \sigma_{j}^{2}}\right] d x
\end{aligned}
$$

and

$$
\begin{aligned}
\frac{\left(e^{a \zeta}-1\right)(1+\zeta)^{3}}{(a \zeta+a-2) e^{a \zeta}+2}=\frac{P}{\sqrt{2} \frac{P}{\pi} V_{0}} \sum_{j=1}^{n} \frac{V_{0 j}}{\sigma_{j}} \int_{0}^{\infty} \frac{(1+x)^{4}}{e^{a x}-1} \\
\times \exp \left[-\frac{\left\{\frac{P(1+x)^{2}}{e^{a x}-1}-\vec{E}_{j}\right\}^{2}}{2 \sigma_{j}^{2}} \mid d x\right.
\end{aligned}
$$

The value of $\zeta$ at a given $P$ is determined as the solution of the above equation by means of an electronic computer. If the partial pulmonary volume is $V_{\zeta}$, in which the stretch $x$ is larger than $\zeta$ or the density of the elastic system is lower than $E_{\zeta}, V_{\zeta}$ is obtained by integration of (20) from $x=\zeta$ to $x=\infty$, instead of that in (20) from $x=0$ to $x=\infty$.

The infinitesimally small transalveolar flow $d \varphi$ in the course of infinitesimally short lapse of time $d t$ is the difference between the volume changes of $V_{\zeta}$ in isobaric and independent expirations in the period. If the pressure increase in isobaric expiration in $d t$ is $d P$, we obtain:

$$
d \varphi=\left|\frac{\partial V_{\zeta}}{\partial P} d P-\frac{\partial V_{\zeta}}{\partial t} d t\right|
$$

or

$$
\frac{d \varphi}{d t}=\left|\frac{\partial V_{\zeta}}{\partial P} \cdot \frac{d P}{d t}-\frac{\partial V_{\zeta}}{\partial t}\right|
$$

This is the differential equation for transalveolar flow $\varphi$. The values for $d P / d t$ at different $P$ is already available in the calculation of (21). The second term of the right side of the equation is written as:

$$
\frac{\partial V_{\zeta}}{\partial t}=-\frac{P}{R_{\zeta}}
$$


if $R_{\zeta}$ is the airway resistance to the pulmonary part $V_{\zeta}$. When $V_{\zeta}$ becomes $V_{0 \zeta}$ in perfect pulmonary relaxation, $R_{\zeta}$ is defined by:

$$
R V_{0}=R_{\zeta} V_{0 \zeta} \text {. }
$$

Consequently, we obtain on the assumption of combined normal distributions of the density of the elastic system:

$$
\begin{aligned}
\frac{1}{R_{\zeta}} & =\frac{V_{0 \zeta}}{R V_{0}} \\
& \left.=\frac{1}{\sqrt{2 \pi} R V_{0}} \sum_{j=1}^{n} \frac{V_{0 j}}{\sigma_{j}} \int_{0}^{E} \zeta \exp _{L}-\frac{\left(E-\bar{E}_{j}\right)^{2}}{2 \sigma_{j}^{2}}\right] d E,
\end{aligned}
$$

in which $E_{\zeta}$ is determined by:

$$
E_{\zeta}=P \frac{(1+\zeta)^{2}}{e^{a \zeta}-1}
$$

\section{Comments}

With (20), (21) and (22) we can establish the fundamental principles of expiratory mechanics on anatomical basis, which are to be applied to individual autopsied lungs. One of the underlying conditions of the derivations is the expiration with the intrinsic contractile force of the lung without participation of thoracic activity. The condition is obviously not realized in living organisms, but it is nevertheless not only necessary but also useful in analytical treatments of pulmonary functions.

Prior to the computations, however, it is required to estimate the parameters comprised in the above expressions. Their estimations will be discussed in a forthcoming report of ours together with the results of computations on individual lungs.

\section{IMPORTANT SYMBOLS}

$x$ : Strain of pulmonary elastic system.

$F$ : Stress of the elastic system at the strain $x$.

$T$ : Tension acting on alveolar septa.

$P$ : Intrapulmonary pressure.

$L_{o}$ : Total length of elastic system in a certain volume of relaxed lungs.

$S_{0}$ : Total surface area of alveolar septa in a certain volume of relaxed lungs.

$V_{v}$ : A certain volume of relaxed lungs. This sign is also used to denote the total lung volume in relaxation.

$L$ : Total length of elastic system in a certain volume of expanded lungs.

$S$ : Total surface area of alveolar septa in a certain volume of expanded lungs.

$V$ : Lung volume in general. 
$E$ : Density of elastic system in relaxed lungs.

$\rho_{0}$ : Total length of elastic system on a unit surface area of alveolar septa in relaxed lungs, or the density of the system on a surface.

$\bar{E}$ : Mean $E$ when the distribution of $E$ is given by a normal distribution.

$\sigma: \quad$ Standard deviation of $E$ under the above condition.

$R$ : Total airway resistance.

$t$ : Time.

$\varphi:$ Transalveolar flow. The volume of air which flows within the lung outside the bronchi.

$i$ : Subscript to denote individual pulmonary portions with different structures.

$j$ : Subscript to denote quantities belonging to different normal distributions.

\section{Acknowledgment}

We are greatly indebted to Drs. T. Takahashi and T. Inawashiro, Computing Center, Tohoku University, for the programming of the calculation.

\section{References}

1) Dayman, H. Mechanics of airflow in health and in emphysema. J. clin. Invest., $1951,30,1175-1190$.

2) Suwa, N., Fukasawa, H., Fujimoto, R. \& Kawakami, M. Strain and stress of pulmonary tissues. Tohoku J. exp. Med., 1966, 90, $61-75$.

3) Comroe, J.H., Jr., Forster, R.E. II., Dubois, A.B., Briscoe, W.A. \& Carlsen, E. The lung, clinical physiology and pulmonary function tests. 2nd ed. 1962, Year Book Medical Publishers, Chicago, p. 178. 